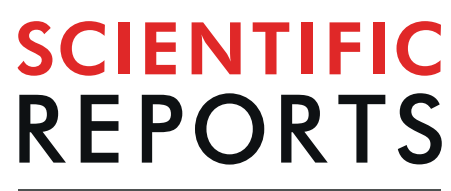

natureresearch

Check for updates

\title{
In-vitro safety and off-target profile of the anti-parasitic arylmethylaminosteroid 10
}

Leonard Blum ${ }^{1,2}$, Sheraz Gul ${ }^{4}{ }^{4}$, Thomas Ulshöfer ${ }^{1}$, Marina Henke ${ }^{1}$, Reimar Krieg ${ }^{5}$, Isabell Berneburg ${ }^{3}$, Dominique Thomas ${ }^{2}$, Sandra Trautmann ${ }^{2}$, Jennifer Kurz ${ }^{1}$, Joachim Geyer $\mathbb{D}^{6}$, Gerd Geisslinger ${ }^{1,2}$, Katja Becker ${ }^{3}$, Michael J. Parnham ${ }^{1}$ \& Susanne Schiffmann ${ }^{1 凶}$

Parasite-mediated diseases like malaria and schistosomiasis are growing health problems worldwide and novel drug candidates are urgently needed. In this study, the in-vitro safety profile of steroid compound 1o (sc10), effective against the parasites Plasmodium falciparum and Schistosoma mansoni with an $\mathrm{IC}_{50}$ value of $5 \mathrm{nM}$, was characterized. We assessed viability/proliferation, apoptosis and cell cycle tests to determine the cytotoxic profile of sc1o in cancer cells. The mutagenic potential was determined with the AMES test. To identify off-target effects we investigated whether sc1o interacts with safety-relevant molecules such as cytochrome P450 (CYP) enzymes, phosphodiesterases (PDE), histone deacteylases (HDAC) and human ether-a-go-go related gene ( $h E R G$ ). Furthermore, to predict the potential bioavailability of sc10, its effect on Caco-2 cell barrier integrity, by measurement of the transepithelial electrical resistance (TEER), was determined. Sc10 at $25 \mu \mathrm{M}$ reduced cell viability, probably through cell-cycle arrest, but did not induce apoptosis in cancer cells. No adverse off-target effects nor mutagenic potential of sc1o were observed. Furthermore, sc1o did not disturb the integrity of the cell barrier, but exhibited low membrane permeability, apparently due to cell adherence. In conclusion, sc1o up to $10 \mu \mathrm{M}$ showed a good in-vitro safety profile.

Parasite mediated diseases such as malaria (Plasmodium) and schistosomiasis (Schistosoma) are growing global health challenges. The WHO reported 228 million cases of malaria worldwide in 2018 (WHO 2018), while schistosomiasis affects approximately $200-250$ million people, mostly in developing countries ${ }^{1-3}$. Since no effective malaria vaccine is available, chemotherapy remains an important weapon against malaria. Standard treatment is commonly based on artemisinin combination therapies, but reports of artemisinin-resistant parasites stress the urgent need for new therapeutic approaches ${ }^{4,5}$. A common therapy approach to schistosomiasis is the use of the anthelminthic drug praziquantel ${ }^{6}$. Frequent use of praziquantel increases the risk of development of resistance mechanisms ${ }^{7,8}$. Therefore, new therapeutic approaches to infections with both parasites are urgently needed.

The steroid compound $1 \mathrm{o}(\mathrm{sclo})$ is a new lead compound with promising activity against intraerythrocytic stages of chloroquine-sensitive and resistant Plasmodium falciparum parasites $\left(\mathrm{IC}_{50} 1-5 \mathrm{nM}\right)^{9}$. Furthermore, in $P$. berghei infected mice, oral administration of sclo drastically reduces parasitaemia and seems to cure the animals ${ }^{9}$. Sclo shows also remarkable activity against the blood-feeding trematode parasite Schistosoma mansoni ${ }^{9}$. With such good activity against the pathogen, an adequate safety and pharmacokinetic profile is crucial for further potential development as a drug candidate. The in-vitro safety profile should include, among others, viability, apoptosis, mitochondrial activity, off-target effects and the AMES test ${ }^{10}$. For the pharmacokinetic profile, the Caco-2 cell barrier assay in-vitro can be used to gain initial insights into the expected bioavailability of the drug ${ }^{11}$. Many drugs are metabolized by cytochrome P450 (CYP) enzymes. This class has more than 50 enzymes, however,

${ }^{1}$ Fraunhofer Institute for Molecular Biology and Applied Ecology IME, Branch for Translational Medicine and Pharmacology (TMP), Theodor-Stern-Kai 7, 60596, Frankfurt/Main, Germany. ${ }^{2}$ pharmazentrum frankfurt/ZAFES, Department of Clinical Pharmacology, Goethe-University Hospital Frankfurt, Theodor-Stern-Kai 7, 60590, Frankfurt/ Main, Germany. ${ }^{3}$ Biochemistry and Molecular Biology, Interdisciplinary Research Center, Justus-Liebig-University, Heinrich-Buff-Ring 26-32, 35392, Giessen, Germany. ${ }^{4}$ Fraunhofer Institute for Molecular Biology and Applied Ecology IME - ScreeningPort, Schnackenburgallee 114, 22525, Hamburg, Germany. ${ }^{5}$ Department of Anatomy II, University Hospital Jena, Teichgraben 7, 07743, Jena, Germany. ${ }^{6}$ Faculty of Veterinary Medicine, Institute of Pharmacology and Toxicology, Justus-Liebig-University, Schubertstraße 81, 35392, Giessen, Germany. ${ }^{\circledR e}$-mail: susanne.schiffmann@ ime.fraunhofer.de 
a

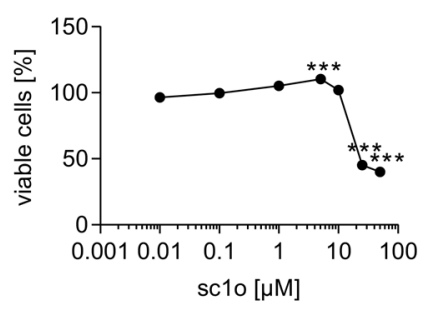

b

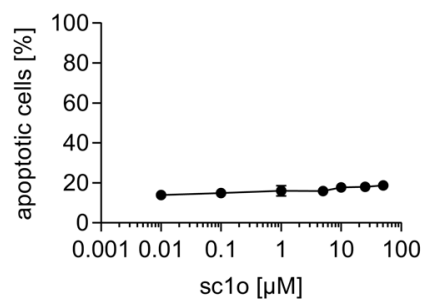

C

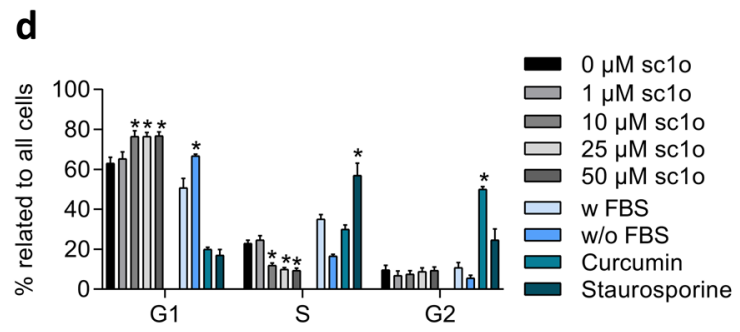

Figure 1. Impact of sclo on cell viability, mitochondrial toxicity and cell cycle. (a) For the cell viability assay, HCT 116 cells were incubated with $0.01-50 \mu \mathrm{M}$ of sclo over $24 \mathrm{~h}$. To calculate cell viability, the absorbance of DMSO-treated cells was set to $100 \%$, and the sclo samples were correlated to the absorbance of DMSO value. (b) For the apoptosis assay, HCT 116 cells were incubated with $0.01-50 \mu \mathrm{M}$ of sclo over $24 \mathrm{~h}$. To calculate the apoptotic rate, the number of apoptotic cells were correlated to the total number of cells. (c) Dose response curves for mitochondrial toxicity by sc1o. The MitoTracker Red CMXRos dye was used to stain mitochondria in live cells. Analysis of mitochondrial toxicity was performed with the Opera imaging system. Sclo was profiled in the assay in 11-point concentration-response format with the raw data normalized using the positive control (1 $\mu \mathrm{M}$ valinomycin) and negative control (DMSO). (d) For cell cycle analysis, HCT 116 cells were incubated with sclo in concentration as indicated or with controls over $24 \mathrm{~h}$. Using flow cytometry, the amount of cells in the various cell cycle phases (G1, S, G2) was determined. The flow cytometry data was analysed with a specific cell cycle analysis method from FlowJo software. For statistical analysis, one-way (a, b) or two-way (d) ANOVA with Dunnett's comparison tests were used. The experiments were performed in triplicate and repeated three times. $* \mathrm{p}<0.05, * * * \mathrm{p}<0.001$ indicate significant differences between sclo and DMSO data.

six of them metabolize 90 percent of drugs, with the three most significant enzymes being CYP3A4, CYP1A2 and CYP2D6 ${ }^{12}$. Therefore, we tested whether sclo interacts with these CYP proteins. To obtain a broad off-target profile of sc1o, further safety-relevant proteins such as the cAMP specific phosphodiesterases (PDE) (PDE4, PDE7, PDE8), histone deacteylases (HDAC) and human ether-a-go-go related gene $(h \mathrm{ERG})^{13}$ were selected. In this current study, these aspects were investigated in cell culture systems to assess whether sclo is a suitable drug candidate for further development.

\section{Results}

Sc10 at high concentrations reduced cell viability in colon cancer cells. A prerequisite for the preclinical characterization of a drug is cytotoxicity testing, which can include proliferation, apoptosis, and cell cycle assays. We investigated whether sclo influences cell viability and proliferation. Hughes et al. recommend for 'no toxicity' concentrations, at least 50 -fold higher values than the $\mathrm{IC}_{50}$ of the test drug ${ }^{10}$. Since sc1o had an $\mathrm{IC}_{50}$ value of about $5 \mathrm{nM}$ on $P$. falciparum ${ }^{9}$, we used a range from $0.01-50 \mu \mathrm{M}$. Cell viability/proliferation was assessed with the WST-1 assay in the colon cancer cell line HCT 116 . At concentrations up to $10 \mu \mathrm{M}$, sclo did not reduce cell viability of HCT 116 cells. However, at $25 \mu \mathrm{M}$ and $50 \mu \mathrm{M}$, sc1o reduced cell viability to about $35 \%$ and $40 \%$, respectively (Fig. 1a).

Sc1o did not induce apoptosis or mitochondrial toxicity. We next investigated whether the reduced cell viability mediated by sclo was due to induction of apoptosis. Caspase 3 activation is a crucial component of the apoptotic machinery ${ }^{14}$. Therefore, apoptosis was detected by activated caspase 3 and staining nuclei (Draq5). HCT 116 cells were incubated with sclo in a range of $0.01-50 \mu \mathrm{M}$ or with DMSO for $24 \mathrm{~h}$. Sc1o and DMSO induced comparable apoptotic rates, indicating that sclo itself did not induce apoptosis in HCT 116 cells (Fig. 1b). Furthermore, we checked whether sclo induces mitochondrial toxicity. Renal carcinoma cells were treated with various concentrations of sclo, and the mitochondrial toxicity was detected using MitoTracker probes, which accumulate only in active mitochondria. However, sclo did not influence the activity of mitochondria (Fig. 1c).

Sc10 at high concentrations induced a G1-block. Another mechanism for reduced cell viability caused by sclo could be the interaction of sclo with the cell cycle. Cell cycle regulation was measured by DNA-staining 
a

\begin{tabular}{cc}
\hline Assay & $\begin{array}{c}\% \text { effect } \\
100 \mu \mathrm{M}[\mathrm{sc1} \text { o] }\end{array}$ \\
\hline HDAC1 inhibition & $9 \pm 1$ \\
\hline HDAC3 inhibition & $9 \pm 0.5$ \\
\hline HDAC6 inhibition & $2 \pm 10$ \\
\hline PDE4A1 inhibition & $0.5 \pm 3$ \\
\hline PDE7A1 inhibition & $4 \pm 6$ \\
\hline PDE8A1 inhibition & $3 \pm 8$ \\
\hline CYP450 3A4 inhibition & $-11 \pm 6$ \\
\hline CYP450 1A2 inhibition & $5 \pm 4$ \\
\hline CYP450 2D6 inhibition & $11 \pm 1$ \\
\hline CYP450 induction (HepG2) & $11 \pm 3$ \\
\hline hERG inhibition & $40 \pm 9$ \\
\hline
\end{tabular}

b

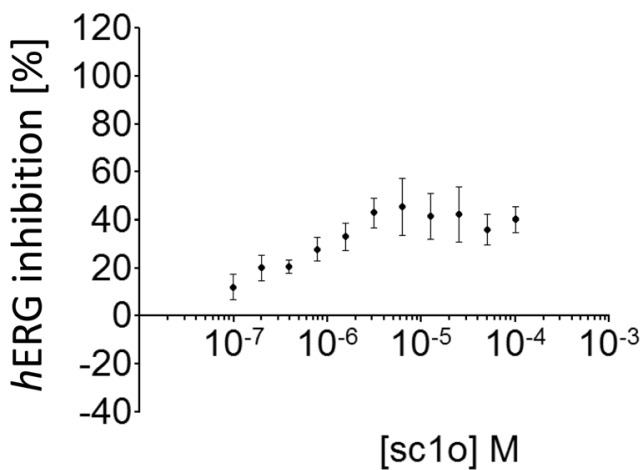

Figure 2. Off-targets effects of sc1o. (a) For the safety profile and off-target studies of sc1o (CYP3A4, CYP1A2, CYP2D6 inhibition, CYP induction, $h$ ERG, HDAC1, HDAC3, HDAC6, PDE4A1, PDE7A1, and PDE8A1), the concentration-response data was fitted to a 4-parameter logistic fit using Prism v5.04 to yield its $\mathrm{IC}_{50}$ in each assay. As sclo was weakly active, the inhibition percentage at $100 \mu \mathrm{M}$ was calculated and reported. (b) Inhibition of $h$ ERG by sclo was calculated using the Predictor $h$ ERG fluorescence polarization assay. As a positive control E-4031, a blocker of $h$ ERG-type potassium channels, yielding $100 \%$ inhibition and as negative control $100 \mathrm{nl}$ of $100 \% \mathrm{v} / \mathrm{v}$ DMSO yielding $0 \%$ inhibition were added.

the cells with propidium iodide and detecting the DNA content and size of the cells via flow cytometry. To analyse the data, the percentages of cells in the different phases (G1, S, and G2) were calculated. As a positive control, G1, $\mathrm{S}$, and G2 blocks were induced with starvation (medium without fetal calf serum (FCS)), $0.2 \mu \mathrm{M}$ of staurosporine, and $20 \mu \mathrm{M}$ of curcumin, respectively (Fig. 1d). Interestingly, $10 \mu \mathrm{M}$ sc1o and higher concentrations also led to a significant increase in cells in the G1 phase and to a significant decrease in cells in the S phase (Fig. 1d). These data indicate that sclo induced a G1 block at higher concentrations $(>10 \mu \mathrm{M})$, a mechanism possibly responsible for the observed reduction in cell viability at these concentrations.

Sc1o did not inhibit off-target enzymes. To further characterize its safety, possible off-target effects of sclo were determined. These studies made use of HDAC1, HDAC3 and HDAC6 assays. Notably, sc1o did not significantly inhibit these HDACs (Fig. 2a). Furthermore, potential effects on PDE4, PDE7 and PDE8 were analysed. Sclo also did not interact with these PDEs (Fig. 2a). Moreover, we analysed whether sclo modulates CYP enzymes (CYP3A4, CYP1A2, CYP2D6). Sc1o did not significantly inhibit CYP enzymes, nor did it mediate CYP induction (Fig. 2a). A moderate inhibition of $h$ ERG function was observed ( $40 \pm 9 \%$ inhibition at $3 \mu \mathrm{M})$ which is within acceptable limits ${ }^{15}$ (Fig. 2a/b). We investigated also whether sclo has mutagenic potential. For this purpose, we tested sc1o in the Salmonella typhimurium strains T100 and T98. To simulate metabolic conversion of sclo by liver enzymes, the compound was incubated with T100 and T98 in the presence of the liver homogenate S9. Sc1o was negative in all AMES mutagenic assays (Fig. 3). These data indicate that sclo has a good in-vitro safety and off-target profile.

No disturbance of cell barrier integrity by sc1o. In multicellular organisms, the epithelial cell layers serve as functional barriers. Key components of epithelial cell barriers are the junctions between adjacent cells. Tight junctions are particularly relevant for the active barrier function of the cell layer, since they regulate the passage of molecules across the barrier by selectively opening and closing in response to various signals from inside and outside the cells. Drugs have to pass through these tissue barriers. A direct correlation exists between the permeability of a cell layer and its electrical resistance, i.e. transepithelial electric resistance (TEER). Therefore, the TEER value can be used to quantify the tightness of the barrier. Additionally, electrical capacitance can be detected, which provides information on the morphology of the membrane, such as the expression of microvilli and other membrane extrusions. Caco- 2 cell barriers were generated on porous membranes, and TEER and capacitance were determined to characterize whether sclo influences the integrity of the cell barrier. Ethylene

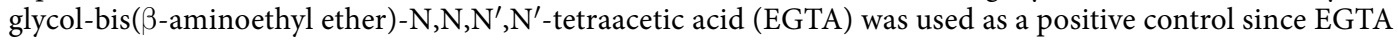
leads to a depletion of extracellular $\mathrm{Ca}^{2+}$, which in turn causes a disassembly of tight junctions ${ }^{16}$. As expected, adding EGTA led to a drop in TEER readings and to an increase in the capacitance (Fig. 4a/b). To quantify the alteration of TEER and capacitance, the TEER/capacitance values $20 \mathrm{~h}$ after adding the various stimuli (EGTA, sc1o) were compared with the TEER/capacitance value before adding the stimuli. EGTA reduced the relative TEER to $0.12 \pm 0.03 \%$ and increased the relative capacitance to $481 \pm 168 \%$ (Fig. $4 \mathrm{c} / \mathrm{d}$ ). Sclo altered neither the TEER nor the capacitance value (Fig. $4 \mathrm{a}-\mathrm{d}$ ).

Low transport rate of sc1o in the Caco-2 cell barrier assay. To determine the permeability of cells to sclo, the amount of sclo that passes through a Caco-2 cell barrier was determined. For this, a Caco-2 cell barrier was generated on a porous membrane and covered with various concentrations of sclo for $24 \mathrm{~h}$. As a control, porous membranes without a cell barrier were incubated with sclo for $24 \mathrm{~h}$. The concentrations of sclo in the 
a

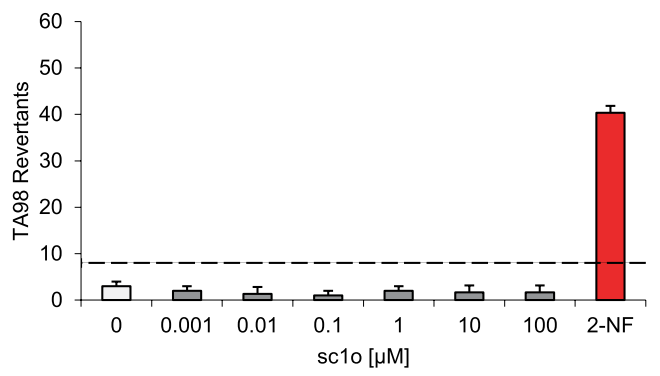

C

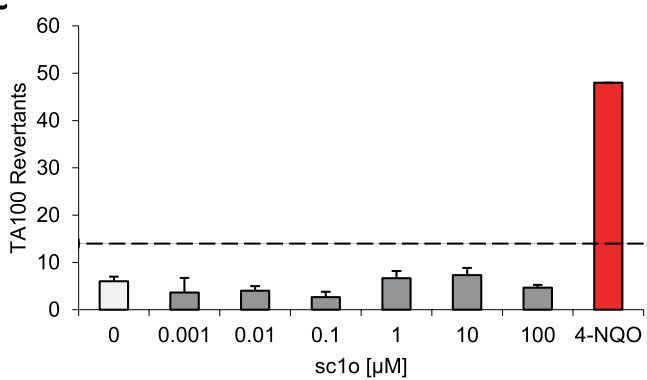

b

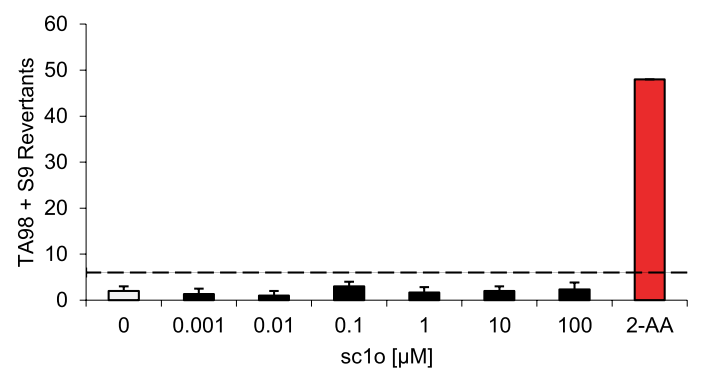

d

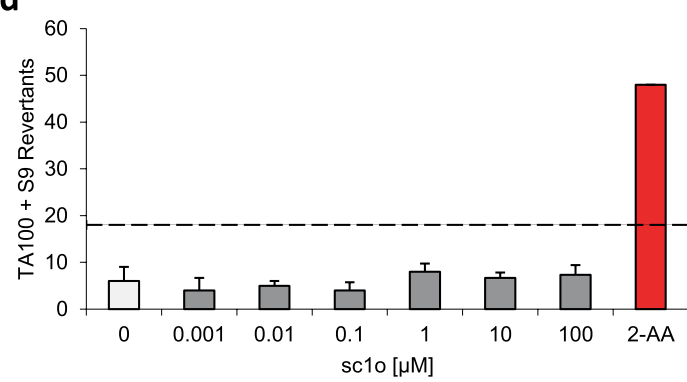

Figure 3. The AMES test reveal no mutagenic potential for sclo. The AMES test was conducted with two Salmonella typhimurium strains TA98 (a/b) and TA100 (c/d) in the presence $(\mathbf{b} / \mathbf{d})$ or absence $(\mathbf{a} / \mathbf{c})$ of liver homogenate S9 to simulate the metabolic conversions of sc1o with liver enzymes. The AMES MPF 98/100 assay from Xenometrix was performed as described by the supplier. Data were analysed with the AMES MPF calculation sheet provided by Xenometrix. The experiment was performed once in triplicates as suggested by the supplier. The dashed line indicate 2 fold increase over baseline. Abb. BL, base line, 2-AA, 2-aminoanthracene; 2-NF, 2-nitrofluorene; 4-NQO: 4-nitroquinoline-N-oxide.

basolateral and apical media were determined using LC-MS/MS. In the apical compartment, sclo concentrations were higher in wells without than in wells with a cell barrier. These data indicate that sclo was bound to the membrane and/or penetrated into the cells (Fig. 4e). Furthermore, in the basolateral compartment, sclo concentrations were higher in wells without a cell barrier than in wells with one, indicating that the cell barrier was functional (Fig. 4f). In the basolateral compartment, a concentration-dependent increase in sclo was detected, indicating that sclo is able to penetrate through the cell barrier (Fig. 4f). However, only one hundredth of the administered $10 \mu \mathrm{M} \mathrm{sclo}$ was found in the basolateral compartment. Bearing in mind that, in the apical compartment, sclo concentration was low in the presence of cells, these findings indicate that the cells absorbed or accumulated sclo in some way. Further studies are necessary to find out whether sclo is bound to the membrane or is taken up by the cell where it can exert activity.

\section{Discussion}

Sclo was recently identified as a new and promising candidate for the treatment of malaria ${ }^{9}$. The next step on the path towards drug development includes generating an in-vitro safety profile and a prediction of potential bioavailability. Our results reveal that sclo did not disturb Caco-2 cell barrier integrity but it is characterized by a low permeability potential. Furthermore, sclo has no off-target effects, no mutagenic potential (AMES test) and no apoptosis induction potential. However, at $>10 \mu \mathrm{M}$, sclo reduced cell viability of HCT 116 cells, possibly by inducing a G1 block, an action linked with reduced cell proliferation in a cancer cell line.

Hughes et al. also stated that cytotoxicity can be considered tolerable if the cytotoxic concentration is at least 50 -fold higher than the effective concentration ${ }^{10}$. We observed cytotoxic effects at $10 \mu \mathrm{M}$ in a cancer cell line, whereas sclo had an $\mathrm{IC}_{50}$ of $4.1 \pm 1.6 \mathrm{nM}$ against the malaria parasite $P$. falciparum (strain 3D7) ${ }^{9}$. Sclo therefore, meets the generally accepted criteria for a lead compound, as reported in the literature ${ }^{10}$, because sclo up to $100 \mu \mathrm{M}$ was largely inactive (yielding $<<50 \%$ inhibition) in all but one of the safety and off-target assays. The only suggestion of a liability detected for sclo was associated with $h$ ERG ( $40 \pm 9 \%$ inhibition at $3 \mu \mathrm{M}$ compound concentration) which is not sufficient to prevent further development of the compound. The G1 block detected in HCT- 116 cells was obtained at $10 \mu \mathrm{M}$ sclo which is also a concentration 2000 times higher than the $\mathrm{IC}_{50}$ (around $5 \mathrm{nM}$ ) for the eradication of $P$. falciparium.

Caco-2 permeability of drugs is a frequently used system to estimate transport across the intestinal epithelium, which is important for drug absorption from the gut. Drugs can use the paracellular and/or the transcellular route to penetrate through the colon wall. For the paracellular route the drug have to migrate through the apical and basolateral membrane and for the transcellular route drugs have to traverse the tight junctions. The Caco- 2 cell barrier assays performed in this study reflect both the para- and the transcellular routes. The effect of sclo on the transcellular route was investigated using the TEER measurements, whereas the trans- and paracellular routes were investigated with the transport assay. Sclo did not alter TEER values, indicating that sclo cannot use the 


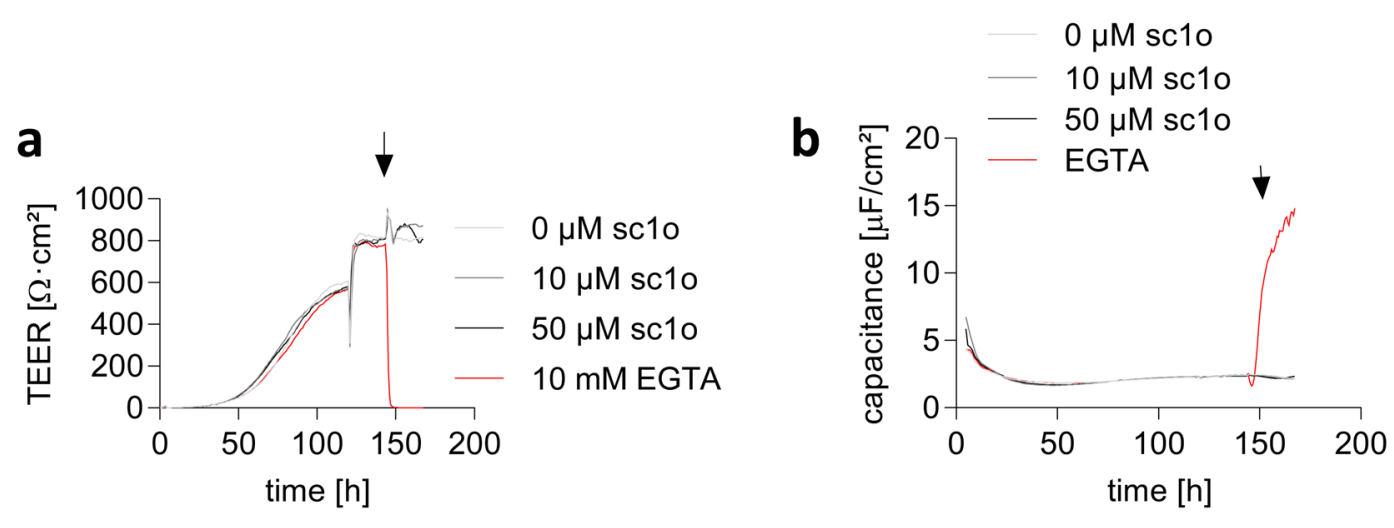

C
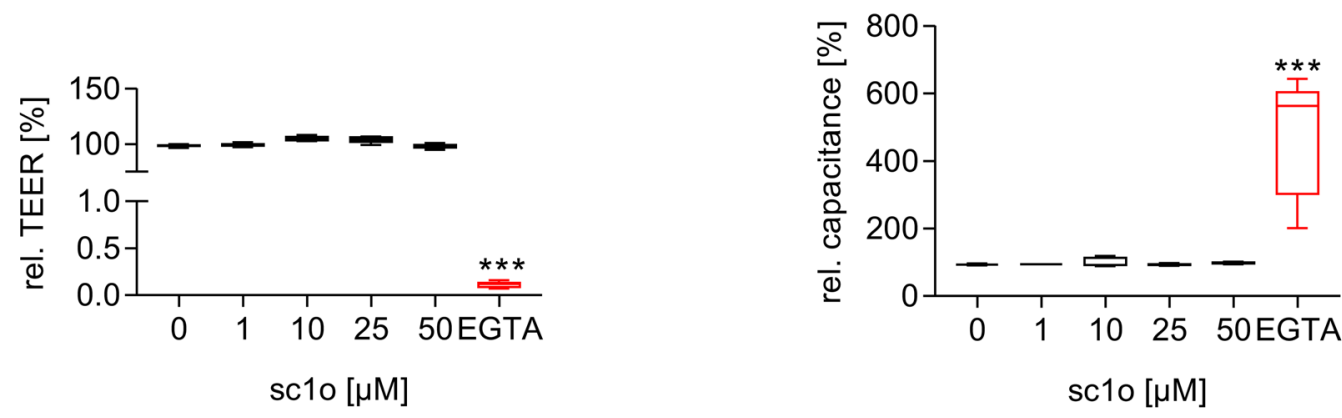

e

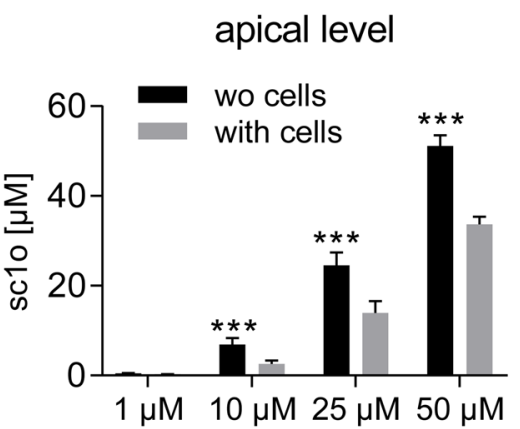

f

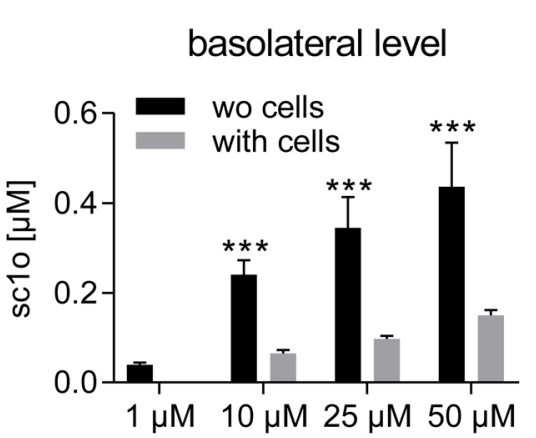

Figure 4. Influence of sc1o on Caco-2 cell barrier integrity and the pass-through rate of sclo. (a/b) TEER (a) and capacitance $(\mathbf{b})$ as a function of time in the presence or absence of different sclo concentrations $(0,10$, $50 \mu \mathrm{M})$ or of $10 \mathrm{mM}$ EGTA. The arrow indicates the addition of buffer containing sclo or EGTA. The data is a representative example from one of three independent experiments performed in duplicate. Caco-2 cells were cultured for 6 days on permeable transwell filters. At day 6, medium with sc1o at various concentrations or EGTA was added. The TEER and capacitance values were followed in a cellZscope 2 device. (c/d) To calculate the effect of sclo on the TEER or capacitance value, the values shortly before adding sclo was set to $100 \%$. The TEER or capacitance values $20 \mathrm{~h}$ after adding the sclo were used to calculate the sclo effects. (e/f). To determine the pass-through rate of sc1o, Caco- 2 cells were cultured for 6 days on permeable transwell filters. On day 6 , medium with sclo at various concentrations was added, and after $24 \mathrm{~h}$, the apical and basolateral medium were collected. As control transwell filters without cell barriers were incubated for $24 \mathrm{~h}$ with the indicated sc1o concentrations. The sclo concentrations in the apical and basolateral medium was determined using LC-MS/ MS. For statistical analysis, two-way ANOVA with Sidak's multiple $(a, c, e, f)$ comparison or one-way analysis and Dunnett's multiple comparisons test $(\mathrm{b}, \mathrm{d})$ was used. $* \mathrm{p}<0.05, * * * \mathrm{p}<0.001$ indicate significant differences between sclo/EGTA and control samples or samples with and without cell barrier.

transcellular route to penetrate trough the colon wall. Sc1o showed also a low transport rate, indicating that it also did not use the transcellular route adequately. The low permeability and possibly high protein binding is in line with a low serum concentration of about $2 \mu \mathrm{M}$ after intraperitoneal administration of $100 \mathrm{mg} / \mathrm{kg} s \mathrm{so}$ in mice . To $^{2}$ improve the bioavailability of sc1o, two options are available. Synthetic modification of the molecular structure and/or suitable formulation, as the absorption of many drug molecules with poor permeability can be improved 
with excipients ${ }^{17}$. For sclo, it has already been shown that the steroid and the hydroxyarylmethylamino moieties are essential for antimalarial activity, supporting a chelate-based quinone methide mechanism involving metal or haem bioactivation ${ }^{9}$. Alteration of the structure is thus, likely to reduce the antimicrobial efficacy. To increase the bioavailability of sclo without reducing its antimicrobial efficacy, a suitable formulation such as solid dispersions, offers many benefits over conventional drug delivery approaches ${ }^{18}$.

Taken together, our findings indicate that sclo has a good in-vitro safety profile and a suitable formulation should ameliorate its low permeability.

\section{Materials and methods}

Cells and reagents. HCT 116 cells were cultured in McCoy's 5 A (modified) medium supplemented with $10 \%$ FCS. RAW264.7 macrophages were cultured in RPMI1640 GlutaMAX medium supplemented with 10\% FCS. HEK293 cells were cultured in DMEM supplemented with GlutaMAX, 10\% heat-inactivated FCS. Caco-2 cells were from Sigma and were cultured in EMEM medium supplemented with $10 \%$ FCS, L-glutamine, and non-essential amino acids (Sigma Aldrich, M7145). All media contain 1\% penicillin/streptomycin, and the cells were cultured at $37^{\circ} \mathrm{C}$ in a $5 \% \mathrm{CO}_{2}$ atmosphere. Sclo was dissolved in DMSO and further diluted in media $\left(c_{\text {stock }}=25 \mathrm{mM}\right.$, maximal DMSO concentration during experiments $\left.0.3 \% \mathrm{v} / \mathrm{v}\right)$. EDTA was from Sigma Aldrich (Schnellendorf, Germany). Lucifer Yellow (sc-215269) was from Santa Cruz. Standard inhibitors included trichostatin A (Sigma-Aldrich, USA), E-4031 (BML-KC158-0005, Enzo Life Sciences, Inc. NY, USA), valinomycin (CAYM10009152-25, Cayman Chemical, Ann Arbor, MI, USA), alpha-naphthoflavone (N5757-1G, SigmaAldrich, USA), quinidine (Q3625-5G, Sigma-Aldrich, USA), ketoconazole (K1003, Sigma-Aldrich, USA), and 3-isobutyl-1-methylxanthine (IBMX) (I7018, Sigma-Aldrich, USA). Human recombinant enzymes included C-ter-His-FLAG-HDAC1 (50051), HDAC-3/NcoR2 (50003) and N-ter-GST-HDAC-6 (50006) from BPS Bioscience (San Diego, CA, USA). PDE4A1 (AMS.60021), and PDE7A1 (AMS.60070) and PDE8A1 (AMS.60080) were purchased from AMSBIO. Assay kits used included Predictor $h$ ERG (Thermo, Waltham, MA, USA), P450Glo (Promega Corp., Madison, WI, USA), and HDAC-Glo Class I/II kits (Promega Corp., Madison, WI, USA).

Cell viability/proliferation assays. For the WST- 1 assays, $2 \times 10^{4} \mathrm{HCT} 116$ cells were incubated for $24 \mathrm{~h}$ at $37^{\circ} \mathrm{C}$. Sc1o $(0-50 \mu \mathrm{M})$ and control (DMSO) were added to the cells and mixed. After a $24 \mathrm{~h}$ incubation step, $10 \mu \mathrm{l}$ WST-1 reagent (Sigma Aldrich, Germany) was added, mixed, and incubated at $37^{\circ} \mathrm{C}$ for $60 \mathrm{~min}$. Absorbance was measured at $450 \mathrm{~nm}$ and at $650 \mathrm{~nm}$ (reference) using an EnSpire plate reader (PerkinElmer, Waltham, MA, USA). The absorbance at $450 \mathrm{~nm}$ was normalized with the absorbance at $650 \mathrm{~nm}$. The sample values were corrected with the background wells (wells with medium and without cells). To calculate cell viability, the absorbance of DMSO-treated cells was set to $100 \%$, and the sc1o samples were correlated to the absorbance of DMSO value.

Apoptosis assay. For this assay, $2 \times 10^{4}$ HCT 116 cells were seeded in a black poly-D-lysine-coated 96-well plate and were incubated for $24 \mathrm{~h}$ at $37^{\circ} \mathrm{C}$. The culture medium was replaced with $100 \mu \mathrm{DMEM}$ medium without phenol red and was supplemented with $10 \%$ FCS and $1 \%$ penicillin/streptomycin. Sc1o $(0-50 \mu \mathrm{M})$ or control (DMSO) were added to the cells and incubated for $24 \mathrm{~h}$ at $37^{\circ} \mathrm{C}$. One $\mu \mathrm{l}$ of CellEvent Caspase- $3 / 7$ green detection reagent (1:10 diluted in DMEM medium without phenol red) was added and incubated for $90 \mathrm{~min}$ at $37^{\circ} \mathrm{C}$ (without $\mathrm{CO}_{2}$ ). Afterwards, $1 \mu$ of DRAQ5 (1:25 diluted in DMEM-medium without phenol red) was added, and the cells were incubated for $30 \mathrm{~min}$ at room temperature (RT). An image was taken using the ImageXpress micro-confocal high-content imaging system (Molecular Device, San Jose, USA). Cell nuclei were stained in red (Cy5 channel); apoptotic cells appeared in green (FITC channel). The percentage of dead cells was determined using the 'live/dead' analysis tool from Molecular Device by calculating the ratio of apoptotic cells (green) to all cells (red). Four different measuring sites in the well were analysed, and the average value was used for data presentation.

Cell cycle assay. For this assay, $2 \times 10^{4} \mathrm{HCT} 116$ were seeded in a 96-well cell culture plate and incubated for $24 \mathrm{~h}$ at $37^{\circ} \mathrm{C}$. The culture medium was replaced with $100 \mu \mathrm{l}$ medium containing sclo in various concentrations or control substances. As a negative control, medium with $10 \%$ FCS was used. G1/S-block was induced via medium without $10 \%$ FCS. Twenty $\mu \mathrm{M}$ curcumin and $0.2 \mu \mathrm{M}$ staurosporine were used to induce G2- and S-block, respectively. After an incubation step of $24 \mathrm{~h}$ at $37^{\circ} \mathrm{C}$, cells were harvested, suspended in $200 \mu \mathrm{l}$ sample buffer $(1 \mathrm{~g}$ glucose/ 11 phosphate-buffered saline (PBS) without calcium or magnesium), mixed, centrifuged ( $200 \mathrm{~g}, 4 \mathrm{~min}$, $4^{\circ} \mathrm{C}$ ), and the supernatant was discarded. This step was repeated once. Cells were fixed with $150 \mu \mathrm{l}$ of ice-cold $70 \%$ ethanol overnight $(>18 \mathrm{~h})$ at $4^{\circ} \mathrm{C}$. Cell pellet was washed with sample buffer, resuspended in $100 \mu$ l staining buffer $(20 \mu / \mathrm{ml}$ propidium iodide and $0.2 \mathrm{mg} / \mathrm{ml}$ RNase in sample buffer) and incubated for $40 \mathrm{~min}$ at RT. Samples were measured within $24 \mathrm{~h}$ in a MACSQuant analyser (Miltenyi Biotec $\mathrm{GmbH}$, Bergisch Gladbach, Germany). Cell cycle distribution was determined using FlowJo software.

Mitochondrial toxicity assay. This assay made use of the MitoTracker Red CMXRos dye (Thermo, Waltham, MA, USA), which stains mitochondria in live cells, and its accumulation is dependent upon membrane potential. The renal carcinoma 786-0 cell line was harvested from a $75 \mathrm{~cm}^{2}$ flask at $80 \%$ confluency by washing it once using $5 \mathrm{ml}$ RT PBS and incubating with $1 \mathrm{ml}$ trypsin $0.05 \%$ / EDTA $0.02 \%$ for $3 \mathrm{~min}$. Cells were suspended in $10 \mathrm{ml}$ pre-warmed cell culture media (RPMI-1640 supplemented with $10 \% \mathrm{FCS}, 100 \mathrm{U} / \mathrm{ml}$ penicillin and $100 \mu \mathrm{g} /$ $\mathrm{ml}$ streptomycin) and counted using a Scepter (Merck Millipore, Germany). Cells were diluted to $7.5 \times 10^{4}$ cells/ $\mathrm{ml}$, and $20 \mu \mathrm{l}$ of this suspension was added to each well of a 384 -well plate. Cells were incubated for $36 \mathrm{~h}$ at $37^{\circ} \mathrm{C}$ and $5 \% \mathrm{CO}_{2}$. Sclo was added using a pre-dilution plate. The positive control was valinomycin at a final concentration of $1 \mu \mathrm{M}$, with the negative control (DMSO) at the same concentration (v/v). After incubation, $10 \mu \mathrm{l}$ of a $200 \mathrm{nM}$ solution of MitoTracker Red CMXRos in pre-warmed cell culture media was added to each well, 
and the cells were incubated for an additional $45 \mathrm{~min}$ at $37^{\circ} \mathrm{C}$ and $5 \% \mathrm{CO}_{2}$. MitoTracker Red CMXRos uptake was measured using an Opera imaging system. To facilitate automatic image analysis, the layout containing the compound area, as well as the valinomycin and DMSO control areas, was created and stored. A sub-layout of five evenly dispersed fields per well was used. These settings also included a measurement height of $1 \mu \mathrm{m}$, which was stored in an exposure file format. By using the stored settings and files, an automated run was repeatedly created and executed. The images obtained were transferred to the file server and uploaded into Columbus 2.4.0 using the built-in helper function and were analysed therein.

HDAC assays. Inhibition of histone deacetylase (HDAC1, HDAC3 and HDAC6) enzymes was measured using the homogeneous, single addition, bioluminogenic HDAC-Glo I/II assay (Promega Corp., USA). Briefly, sclo in 11-point concentration-response format in triplicate ( $100 \mathrm{nl}$ of $1 \mathrm{mM}$ solution in $100 \% \mathrm{v} / \mathrm{v} \mathrm{DMSO}$ ), positive controls (trichostatin A, final concentration of $1 \mu \mathrm{M}$ and $1 \% \mathrm{v} / \mathrm{v}$ DMSO) yielding $100 \%$ inhibition, and negative controls ( $100 \mathrm{nl}$ of $100 \% \mathrm{v} / \mathrm{v}$ DMSO) yielding $0 \%$ inhibition were added to each well of a 384-well microtitre plate by using the Echo 550 liquid handler. Assays were initiated by adding $10 \mu \mathrm{l} /$ well of the HDAC-Glo I/II assay reagent (prepared by rehydrating lyophilized HDAC-Glo I/II substrate in $10 \mathrm{ml}$ HDAC-Glo I/II assay buffer and $10 \mu \mathrm{l}$ developer reagent) and were mixed briefly via orbital shaking (500-700 rpm). The luminescence was measured at steady-state signal:background, which was achieved after 20 min incubation at RT using an EnVision Multilabel 2103 reader (PerkinElmer, Waltham, MA, USA).

PDE assays. Inhibition of phosphodiesterase (PDE4A1, PDE7A1 and PDE8A1) enzymes was measured using the LANCE Ultra CAMP assay (PerkinElmer, Waltham, MA, USA). Briefly, sc1o in 11-point concentration-response format in triplicate $(100 \mathrm{nl}$ of $1 \mathrm{mM}$ solution in $100 \% \mathrm{v} / \mathrm{v}$ DMSO), positive controls (IBMX with final concentration of $100 \mu \mathrm{M}$ and $1 \% \mathrm{v} / \mathrm{v}$ DMSO) yielding $100 \%$ inhibition and negative controls $(100 \mathrm{nl}$ of $100 \% \mathrm{v} / \mathrm{v}$ DMSO) yielding $0 \%$ inhibition were added to each well of a 384 -well microtitre plate by using the Echo 550 liquid handler. Ten $\mathrm{nM}$ of cAMP solution in assay buffer $(5 \mu \mathrm{l} /$ well) was subsequently added and incubated for $45 \mathrm{~min}$ at RT. This was followed by Eu-cAMP tracer in detection buffer (to stop the enzymatic reaction), including $2 \mathrm{mM}$ of IBMX ( $5 \mu \mathrm{l} /$ well $)$ and ULight-anti-cAMP in detection buffer ( $5 \mu \mathrm{l} /$ well), and was incubated for $1 \mathrm{~h}$ at RT, after which the TR-FRET signal was measured using an EnVision Multilabel 2103 reader (PerkinElmer, Waltham, MA, USA).

CYP assays. The inhibition of CYP (3A4, 1A2 and 2D6) and induction of CYP were measured using the luminescence-based P450-Glo (Promega Corp., USA) assay system. Briefly, sc1o in 11-point concentration-response format in triplicate $(100 \mathrm{nl}$ of $1 \mathrm{mM}$ solution in $100 \% \mathrm{v} / \mathrm{v}$ DMSO), positive controls (3A4, ketoconazole; 1A2, $\alpha$-naphthoflavone; 2D6, quinidine with a final concentration of $1 \mu \mathrm{M}$ and $1 \% \mathrm{v} / \mathrm{v}$ DMSO) yielding $100 \%$ inhibition, and negative controls ( $100 \mathrm{nl}$ of $100 \% \mathrm{v} / \mathrm{v}$ DMSO) yielding $0 \%$ inhibition were added to each well of a 384 -well microtitre plate by using the Echo 550 liquid handler. This was followed by adding the $\mathrm{CYP} /$ substrates $(5 \mu \mathrm{l} /$ well $)$ and was incubated for $30 \mathrm{~min}$ at $37^{\circ} \mathrm{C}$. Reactions were initiated by adding an NADPH regeneration system $(5 \mu \mathrm{l} /$ well). The reactions were stopped by adding luciferin detection reagent $(10 \mu \mathrm{l} /$ well), followed by an additional $30 \mathrm{~min}$ incubation at $37^{\circ} \mathrm{C}$ with the luminescence signal detected using an Infinite M1000 PRO plate reader (Tecan, Männedorf, Switzerland).

$h$ ERG assay. Inhibition of $h$ ERG was measured using the Predictor $h$ ERG fluorescence polarisation assay (Thermo Fisher Scientific, Waltham, MA, USA). Briefly, sclo in 11-point concentration-response format in triplicate ( $100 \mathrm{nl}$ of $1 \mathrm{mM}$ solution in 100\% v/v DMSO), positive controls (E-4031, a blocker of $h$ ERG-type potassium channels) yielding 100\% inhibition, and negative controls (100 $\mathrm{nl}$ of $100 \% \mathrm{v} / \mathrm{v}$ DMSO) yielding $0 \%$ inhibition were added to each well of a 384-well microtitre plate by using the Echo 550 liquid handler. This was followed by adding homogenised membrane solution $(5 \mu \mathrm{l} /$ well $)$ and a $1 \mathrm{nM}$ final concentration in assay $(5 \mu \mathrm{l} /$ well). The plates were incubated for $2 \mathrm{~h}$ at RT in a humidity-controlled incubator, and fluorescence polarisation was measured using an EnVision Multilabel 2103 reader (PerkinElmer, Waltham, MA, USA).

AMES test. The AMES test was conducted with two Salmonella typhimurium strains TA98 and TA100 in the presence or absence of liver homogenate S9 to simulate the metabolic conversions of sclo with liver enzymes. The Ames MPF 98/100 from Xenometrix was used as described by the supplier. The principle of the test is based on mutated bacteria strains. Point mutations were made in the histidine operon of Salmonella typhimurium, rendering the bacteria incapable of producing the corresponding amino acid. These mutations resulted in histidine-deficient organisms that cannot grow unless histidine is supplied. When a mutagenic event occurs, base substitutions or frameshifts within the gene may cause a reversion to amino acid prototrophy. These reverted bacteria will then grow in histidine-deficient media. After exposure with increasing concentrations of sclo or with positive controls $(2 \mu \mathrm{g} / \mathrm{ml}$ for $2-\mathrm{NF}$ (TA98), $0.1 \mu \mathrm{g} / \mathrm{ml}$ for $4-\mathrm{NQO}$ (TA100), $2.5 \mu \mathrm{g} / \mathrm{ml}$ (TA100), and $1.0 \mu \mathrm{g} /$ $\mathrm{ml}$ (TA98) for 2-AA red, the cultures were diluted in $\mathrm{pH}$ indicator medium lacking histidine and aliquoted into a 384-well plate. Within two days, cells that had undergone reversion to amino acid prototrophy grew into colonies. Bacterial metabolism reduces the $\mathrm{pH}$ of the medium, changing the color of that well. Finally, the number of wells containing revertant colonies were counted for each dose and compared to a solvent (negative) control. The experiment was conducted once in triplicate. The data was analysed with the AMES MPF calculation sheet provided by Xenometrix. Fold induction over the baseline was the ratio of the mean number of positive wells for the dose concentration divided by the baseline. The baseline is obtained by adding one standard deviation to the mean number of positive wells of the solvent control. Compounds with mutagenic potential are characterized by revertant numbers above the baseline 
Cell barrier model. Twenty thousand CaCo-2 cells were seeded on 24-well ThinCerts (pre-coated with FCS for $30 \mathrm{~min}$ ). In the lower compartment (basolateral), $1 \mathrm{ml}$ culture medium per well was added. As a control, half of the ThinCerts were not seeded with cells. The ThinCerts were transferred to the cellZscope2 (nanoAnalytics). Two hundred $\mu \mathrm{l}$ of medium was added to the ThinCerts to reach a final volume of $300 \mu \mathrm{l}$ in the upper compartment (apical). The TEER and capacitance were measured every hour. After 5 days, the TEER reached a constant value of 700-900 $\mathrm{Ohm}^{*} \mathrm{~cm}^{2}$ and the medium was replaced with EMEM with $2 \% \mathrm{FCS}, 2 \mathrm{mM} \mathrm{L}$-glutamine, $1 \mathrm{x}$ non-essential amino acids, and $110 \mathrm{nM}$ hydrocortisone. The TEER and capacitance were measured every hour for 1 day. Sc1o $(0 \mu \mathrm{M}, 1 \mu \mathrm{M}, 10 \mu \mathrm{M}, 25 \mu \mathrm{M}, 50 \mu \mathrm{M})$ diluted in EMEM with $2 \%$ FCS, 2 mM L-glutamine, $1 \mathrm{x}$ non-essential amino acids, and $110 \mathrm{nM}$ hydrocortisone were added to the apical compartment. As a control, $10 \mathrm{mM}$ of EGTA was used. The cellZscope 2 module was transferred into the incubator for $24 \mathrm{~h}$, and every $30 \mathrm{~min}$ the TEER and capacitance were detected. TEER and capacitance values at the time point of adding the compound solution were used as references to calculate the alteration ( $\mathrm{t} 0 \mathrm{~h}$-value). For the determination of the transport rate the Caco- 2 cell barrier was generated as mentioned above. Sc1o $(0 \mu \mathrm{M}, 1 \mu \mathrm{M}, 10 \mu \mathrm{M}, 25 \mu \mathrm{M}, 50 \mu \mathrm{M})$ diluted in EMEM with 2\% FCS, $2 \mathrm{mM}$ L-glutamine, $1 \mathrm{x}$ non-essential amino acids, and $110 \mathrm{nM}$ hydrocortisone were added to the apical compartment and shaked at $40 \mathrm{rpm}$. At time point $0 \mathrm{~h}$ and after $24 \mathrm{~h}$ the medium of the basolateral and apical compartment was collected and stored at $-20^{\circ} \mathrm{C}$ and analysed by LC-MS/MS.

Determination of sc1o via LC-MS/MS. For the quantification of sclo, $20 \mu$ samples were mixed with $20 \mu \mathrm{l}$ acetonitrile, $20 \mu \mathrm{l}$ of the internal standard ( $\mathrm{sclc}, 200 \mathrm{ng} / \mathrm{ml}$ in acetonitrile) and $150 \mu \mathrm{l}$ methanol. The mixture was vortexed for $1 \mathrm{~min}$ and centrifuged at 20,000 $\mathrm{g}$ for $3 \mathrm{~min}$, and the clear supernatant was transferred to an autosampler vial. Samples in which a high concentration of sclo was expected were diluted by a factor of 50 with $25 \%$ DMSO in water, and $20 \mu \mathrm{l}$ of the diluted sample was processed as stated. For calibration and quality control samples, $20 \mu \mathrm{l}$ water were mixed with $20 \mu \mathrm{l}$ standard or quality control working solution and processed as stated. The amount of sclo was analysed via liquid chromatography coupled to tandem mass spectrometry. An Agilent 1260 series binary pump (Agilent technologies, Waldbronn, Germany) equipped with a Zorbax Eclipse Plus C18 UHPLC column ( $50 \mathrm{~mm} \times 2.1 \mathrm{~mm}$ ID, $1.8 \mu \mathrm{m}$, Agilent technologies, Waldbronn, Germany) was used for chromatographic separation. Mobile phase A was water with $0.2 \%$ formic acid and $10 \mathrm{mM}$ ammonium formate, whereas mobile phase B was acetonitrile/isopropanol/acetone (50:30:20, v/v/v) with $0.2 \%$ formic acid. The gradient program started with $65 \%$ mobile phase A for $0.5 \mathrm{~min}$, then A was decreased within $1.5 \mathrm{~min}$ to $0 \%$, held at $0 \%$ for $1 \mathrm{~min}$ and increased to $65 \%$ again within $0.1 \mathrm{~min}$. Total run time was $5 \mathrm{~min}$. The MS/MS analyses were performed using a triple quadrupole mass spectrometer QTrap 5500 (Sciex, Darmstadt, Germany) equipped with a Turbo $\mathrm{V}$ ion source operating in positive electrospray ionization mode. The analysis was done in multiple reaction monitoring (MRM) mode. Information on the recorded mass transitions for the analyte and internal standard is given in Supplemental Fig. 1. Data was acquired using Analyst Software V 1.6.2 and quantified with MultiQuant Software V 3.0.2 (both Sciex, Darmstadt, Germany), employing the internal standard method (isotope dilution mass spectrometry). The calibration curve was calculated via linear regression with $1 / \mathrm{x}$ weighting. Variations in the accuracy of the calibration standards were less than $15 \%$ over the whole range of calibration, except for the lower limit of quantification where a variation in accuracy of $20 \%$ was accepted.

Statistics. Results are presented as means \pm standard errors (SEM). The data was analysed with one-way or two-way analysis of variance (ANOVA) and with Dunnet's or the Shapiro-Wilk comparison test. For all calculations and creation of graphs, GraphPad Prism 8 was used and $p<0.05$ was considered the threshold for significance. For the safety profile and off-target studies of sc1o (mitochondrial toxicity, CYP 3A4, CYP 1A2, CYP 2D6 inhibition, CYP induction, $h$ ERG, HDAC1, HDAC3, HDAC6, PDE4A1, PDE7A1, and PDE8A1), the concentration-response data was fitted to a 4-parameter logistic fit using Prism v5.04 to yield its $\mathrm{IC}_{50}$ in each assay. As sclo was weakly active, the inhibition percentage at $100 \mu \mathrm{M}$ was calculated and reported.

\section{Data availability}

The data from any performed experiment is available from the corresponding author.

Received: 28 January 2020; Accepted: 15 April 2020;

Published online: 05 May 2020

\section{References}

1. Steinmann, P., Keiser, J., Bos, R., Tanner, M. \& Utzinger, J. Schistosomiasis and water resources development: systematic review, meta-analysis, and estimates of people at risk. Lancet Infect Dis 6, 411-425, https://doi.org/10.1016/S1473-3099(06)70521-7 (2006).

2. Anisuzzaman \& Tsuji, N. Schistosomiasis and hookworm infection in humans: Disease burden, pathobiology and anthelmintic vaccines. Parasitol Int 75, 102051, https://doi.org/10.1016/j.parint.2020.102051 (2020).

3. WHO. Schistosomiasis. Fact sheet $\mathrm{N}^{\circ} 115$ (2015). (2015).

4. Sinha, S., Medhi, B. \& Sehgal, R. Challenges of drug-resistant malaria. Parasite 21, 61, https://doi.org/10.1051/parasite/2014059 (2014).

5. Wells, T. N., Hooft van Huijsduijnen, R. \& Van Voorhis, W. C. Malaria medicines: a glass half full? Nat Rev Drug Discov 14, 424-442, https://doi.org/10.1038/nrd4573 (2015)

6. Utzinger, J. \& Keiser, J. Schistosomiasis and soil-transmitted helminthiasis: common drugs for treatment and control. Expert Opin Pharmacother 5, 263-285, https://doi.org/10.1517/14656566.5.2.263 (2004).

7. Botros, S. S. \& Bennett, J. L. Praziquantel resistance. Expert Opin Drug Discov 2, S35-40, https://doi.org/10.1517/17460441.2.S1.S35 (2007).

8. Mader, P., Rennar, G. A., Ventura, A. M. P., Grevelding, C. G. \& Schlitzer, M. Chemotherapy for Fighting Schistosomiasis: Past, Present and Future. ChemMedChem 13, 2374-2389, doi:10.1002/cmdc.201800572 (2018).

9. Krieg, R. et al. Arylmethylamino steroids as antiparasitic agents. Nat Commun 8, 14478, https://doi.org/10.1038/ncomms14478 (2017). 
10. Hughes, J. P., Rees, S., Kalindjian, S. B. \& Philpott, K. L. Principles of early drug discovery. Br J Pharmacol 162, 1239-1249, https:// doi.org/10.1111/j.1476-5381.2010.01127.x (2011).

11. Hubatsch, I., Ragnarsson, E. G. \& Artursson, P. Determination of drug permeability and prediction of drug absorption in Caco-2 monolayers. Nat Protoc 2, 2111-2119, https://doi.org/10.1038/nprot.2007.303 (2007).

12. Lynch, T. \& Price, A. The effect of cytochrome P450 metabolism on drug response, interactions, and adverse effects. Am Fam Physician 76, 391-396 (2007)

13. Kalyaanamoorthy, S. \& Barakat, K. H. Development of Safe Drugs: The hERG Challenge. Med Res Rev 38, 525-555, https://doi. org/10.1002/med.21445 (2018).

14. Porter, A. G. \& Janicke, R. U. Emerging roles of caspase-3 in apoptosis. Cell death and differentiation 6, 99-104, https://doi. org/10.1038/sj.cdd.4400476 (1999).

15. Moraes, C. B. et al. Accelerating Drug Discovery Efforts for Trypanosomatidic Infections Using an Integrated Transnational Academic Drug Discovery Platform. SLAS Discov 24, 346-361, https://doi.org/10.1177/2472555218823171 (2019).

16. Rothen-Rutishauser, B., Riesen, F. K., Braun, A., Gunthert, M. \& Wunderli-Allenspach, H. Dynamics of tight and adherens junctions under EGTA treatment. J Membr Biol 188, 151-162, https://doi.org/10.1007/s00232-001-0182-2 (2002).

17. Sharma, P., Varma, M. V., Chawla, H. P. \& Panchagnula, R. Absorption enhancement, mechanistic and toxicity studies of medium chain fatty acids, cyclodextrins and bile salts as peroral absorption enhancers. Farmaco 60, 884-893, https://doi.org/10.1016/j. farmac.2005.08.008 (2005).

18. Paudwal, G. et al. Recent Advances in Solid Dispersion Technology for Efficient Delivery of Poorly Water-Soluble Drugs. Curr Pharm Des 25, 1524-1535, https://doi.org/10.2174/1381612825666190618121553 (2019).

\section{Acknowledgements}

This work was supported by the Landesoffensive zur Entwicklung wissenschaftlich-ökonomischer Exzellenz (LOEWE) Research Centre for Translational Medicine and Pharmacology (TMP), the LOEWE Research Centre for Novel Drug Targets against Poverty-Related and Neglected Tropical Infectious Diseases (DRUID) and the LOEWE Centre for Translational Biodiversity Genomics (TBG).

\section{Author contributions}

L.B.: Conceptualization, Investigation, Data curation, Writing - original draft, Writing - revision. S.G.: Investigation, Writing - original draft. T.U.: Investigation. M.H.: Investigation. R.K.: Investigation. I.B.: Investigation. D.T.: Investigation. S.T.: Investigation. J.K.: Investigation. J.G.: Conceptualization. G.G.: Conceptualization. K.B.: Conceptualization. M.J.P.: Conceptualization, Writing - original draft, Writing revision. S.S.: Conceptualization, Data curation, Writing - original draft, Writing - revision.

\section{Competing interests}

The authors declare no competing interests.

\section{Additional information}

Supplementary information is available for this paper at https://doi.org/10.1038/s41598-020-64382-w.

Correspondence and requests for materials should be addressed to S.S.

Reprints and permissions information is available at www.nature.com/reprints.

Publisher's note Springer Nature remains neutral with regard to jurisdictional claims in published maps and institutional affiliations.

Open Access This article is licensed under a Creative Commons Attribution 4.0 International License, which permits use, sharing, adaptation, distribution and reproduction in any medium or format, as long as you give appropriate credit to the original author(s) and the source, provide a link to the Creative Commons license, and indicate if changes were made. The images or other third party material in this article are included in the article's Creative Commons license, unless indicated otherwise in a credit line to the material. If material is not included in the article's Creative Commons license and your intended use is not permitted by statutory regulation or exceeds the permitted use, you will need to obtain permission directly from the copyright holder. To view a copy of this license, visit http://creativecommons.org/licenses/by/4.0/.

(c) The Author(s) 2020 